\title{
Cell sorting based on motility differences
}

\author{
Carine P. Beatrici ${ }^{*}$ and Leonardo G. Brunnet ${ }^{\dagger}$ \\ Instituto de Física, Universidade Federal do Rio Grande do Sul, Av. Bento Gonçalves, 9500, P.B. 15051, 91501 -970 Porto Alegre, Brazil
}

(Received 10 January 2011; revised manuscript received 26 August 2011; published 27 September 2011;

publisher error corrected 3 October 2011)

\begin{abstract}
Self-propelled particles are used to simulate cell aggregates in a model considering homogeneous adhesion forces between cells and using only motility differences as segregation drivers. The tendency of cells to follow their neighbors is also included in the formulation. Three model variants are explored, and the conditions on which motility differences may produce segregation are mapped in parameter diagrams. The evolution of the order parameter measuring cell segregation is similar to those found by models based on differential adhesion. It is also found that, considering only velocity differences, the faster cells envelope the slower ones, which is opposite to the ordering observed in early experiments by Jones and co-workers [Jones, Evans, and Lee, Exp. Cell. Res. 180, 287 (1989)].
\end{abstract}

DOI: 10.1103/PhysRevE.84.031927

PACS number(s): 87.17.-d, 36.40.Ei

\section{INTRODUCTION}

Complex organisms have generally low capacity to regenerate and may recover only small parts of their bodies. However, some simple living beings can reconstruct their bodies even when their cells are dispersed and randomly mixed. Starting from this state they can return to the original structures in a process called cell sorting. Studying segregation in simple beings with two tissues is the first step in understanding how cells organize during a regeneration process. Such a study explores an important threshold in the evolution of species: the evolutionary transition from unicellular to multicellular organisms. Knowledge of this segregation process can also help us to understand regeneration in more complex beings.

Cell sorting is an old problem with the first studies dating from the 1740s. Trembley (cited in Ref. [1]) cut a hydra in two and, a few hours later, found two hydras regenerated from the original one. Since then, the hydra has been one of the most common model organisms used to study regeneration [2]. An important experimental step was given when Schiefferdecker [3] succeeded in separating two living tissues using a pancreatic extract. In 1907 Wilson [4] did the first cell-sorting experiment, when he forced the cells of a sponge through a sieve, separating them. After immersion in sea water, they eventually agglomerated and formed a whole new sponge. He also mixed cells of different sponge species, but they regenerated correctly, preserving the cells of their own species. In 1944 Holtfreter [5] performed again the experiment of Wilson with amphibian cells, and, since cells of different tissues presented different pigmentation, he verified that they diffused during the segregation process, discarding the hypothesis of cytological transformation.

The differential adhesion hypothesis (DAH) was proposed by Steinberg [6] in 1963 to explain cell sorting. According to this approach, cells of different tissues have different degrees of adherence, and the more adhesive cells tend to aggregate in a central cluster with the less adhesive cells around the center. An alternative hypothesis based on recognition mechanisms and velocity differences was experimentally tested by Jones,

\footnotetext{
*carine.beatrici@ufrgs.br

${ }^{\dagger}$ leon@if.ufrgs.br
}

Evans, and Lee, published in 1989 [7]. They ascribed the reorganization of cells, at least in part, to differences in motility. In their words: "Since this positioning ultimately depends on the ability of cells to translocate within the aggregate matrix, it seemed reasonable to speculate that differences in cell motility may be responsible for the orientation of cells of different type into the inside or outside positions." In the experiment they found that the faster migrating cells present inside positioning in heterotypic aggregates.

We can simulate these different hypotheses using mathematical models found in the literature: the cellular Potts model approach [8-10] and boids-like approach [11]. Both have already been used to study cell sorting under the DAH, but neither of these approaches has explored whether cell sorting may be caused only by motility differences. So the aim of this work is to test if the differential velocity hypothesis is a possible physical mechanism for cell segregation. If positive, the basic question about inside or outside cell positioning and the need for further hypothesis should also be answered. In fact, as will be shown in this work, although motility differences alone may lead to sorting under adequate conditions, this sorting leaves slower cells surrounded by faster cells, which is the opposite of the ordering observed in the experiments cited above. In addition, we also look for the scale relations describing the segregation evolution and the range of parameters where cell sorting under this mechanism may exist.

\section{THE MODEL}

The boids model was proposed by Reynolds in 1987 [12]. His objective was to create a model describing the coordinated motion of bird flocks or fish schools [12]. A systematic study of the parameter space of a two-dimensional boids model was presented by Vicsek et al. [13]. They proposed a simple system and showed that, above a critical density, there is a transition to an ordered moving state. In this model the position of a particle is given by

$$
\vec{x}_{i}(t+1)=\vec{x}_{i}(t)+\vec{v}_{i}(t) \Delta t .
$$

The speed $\vec{v}_{i}(t)$ in Eq. (1) has constant module $v_{0}$, and the direction each particle (boid) takes results from an average 
over directions of neighboring boids and a noise term. In this work we use a two-dimensional system with boids to represent cells, so we add terms to account for their volume, for the local attractiveor repulsive forces $[11,14]$, and a coordinated motion term to represent their tendency to follow the others $[15,16]$ :

$$
\theta_{i}(t+1)=\arg \left[\alpha \sum_{j\langle\mathrm{viz}\rangle} \frac{\vec{v}_{j}(t)}{v_{0}}+\beta \sum_{j} \vec{f}_{i j}(t)+\eta \vec{u}_{i}(t)\right] .
$$

In this expression, $\alpha$ is the parameter regulating the coordinated motion; $\vec{v}_{j}$ is the velocity of a neighbor cell $j ; \beta$ is the intensity of the force between cells; $\vec{f}_{i j}$ expresses the dependency of the force on the distance between cells $i$ and $j$; and $\vec{u}$ is a unitary noise vector pointing in a random direction, where its intensity is determined by $\eta$.

Cells within the force range are considered to be neighbors. The force $\vec{f}_{i j}$ is composed of three parts: For distances shorter than a core radius $r_{c}$, the force is infinite (repulsive) characterizing the cell volume; for distances $r_{c}<r<r_{0}$ around and equilibrium distance $r_{e}$, the force is harmonic-like; and for distances greater than $r_{0}$ the force is null:

$$
\vec{f}_{i j}=\vec{e}_{i j} \begin{cases}+\infty ; & r_{i j} \leqslant r_{c} \\ 1-\frac{r_{i j}}{r_{e}} ; & r_{c}<r_{i j}<r_{0} \\ 0 ; & r_{i j} \geqslant r_{0},\end{cases}
$$

where $\vec{e}_{i j}$ is an unit vector oriented from particle $i$ to $j$, and $r_{i j}$ is the distance between cells $i$ and $j$.

Here we simulate a diploblastic organism composed by two cell types: endodermic and ectodermic. The velocity difference mechanism in Eq. (1) is introduced ascribing speeds $v_{0}$ and $v_{1}$ to the two different kinds of cells. Their speed ratio defines parameter $\delta\left(\equiv v_{1} / v_{0}\right)$. In this work we have used $r_{c}=0.2$, $r_{e}=0.4, r_{0}=0.55, \eta=1$, and a time step $\Delta t=1$. In order to avoid boids displacement larger than cell dimensions at each time step, the faster cells' speed is limited to a maximum of $v \leqslant 0.03$, while the slower cells' speed is fixed at $v_{0}=0.007$. In the simulations we have to deal with the infinite forces defined in Eq. (3). Since the intensity of forces out of the cell core is on the order of 1 , a value of $10^{5}$ (instead of infinite) is large enough to guarantee that, if two cells have any core superposition, they will move in opposite directions.

A typical two-dimensional cell aggregation experiment is constructed using previously dissociated ectodermic and endodermic cells sandwiched between glass slides separated by the distance of a single cell layer occupying an area of $\sim$ $250 \mu \mathrm{m}$ of radius with approximately 1000 cells [17]. Cells of different tissues are initially completely mixed. After evolving along 6 hours, their positioning is rearranged to a segregated state where endodermic cells end up completely surrounded by ectodermic cells. Cells diffuse at approximately $1 \mu \mathrm{m}^{2} / \mathrm{min}$, with endo-ecto diffusion more than two times faster than endo-endo [18]. Given the experimental typical time to achieve the segregated state and the typical diffusion speed, we can establish a time scale for the simulation time step. Taking $\sim 10 \mu \mathrm{m}$ for cell diameters, a value of $\sim 100$ timesteps $/ \mathrm{s}$ in the simulation would result in realistic values for the diffusion and for the segregation times.
The presence of velocity differences also implies redefining the first term in Eq. (2), since it is velocity dependent. So we rewrite this equation in the following way:

$$
\theta_{i}(t+1)=\arg \left[\sum_{j\langle\mathrm{viz}\rangle} \alpha_{i j} \frac{\vec{v}_{j}(t)}{v_{0}}+\beta \sum_{j} \vec{f}_{i j}(t)+\eta \vec{u}_{i}(t)\right] .
$$

Distinct approaches may be derived from Eq. (4); we have chosen three limiting cases: (1) model $\mathrm{A}$-the same coordinated motion parameter is used for all particles $\alpha_{i j}=\alpha$; (2) model B-parameter $\alpha_{i j}$ will depend only on particle $i$, $\alpha_{i}=\alpha / \delta_{i}$; and (3) model C-parameter $\alpha_{i j}$ will depend on the neighboring particles $j, \alpha_{i j}=\alpha / \delta_{j}$. In model A both kinds of cells tend to align preferentially with the faster ones than with the slower. In model B the influence of the neighboring particles' movement is stronger in the slower particles than in the faster. In model $\mathrm{C}$ all cells are equally favored by the alignment term. So the coordinated movement weight relative to adhesion and noise differs in these three models; as will be shown, these differences have important implications in the parameter space available for cell segregation.

In order to evaluate the spatial organization during the cell-sorting process, we define a parameter that measures the average fraction of neighbors with velocity $v_{1}$ around a given cell with velocity $v_{0}[11]$ :

$$
\gamma=\left\langle\frac{n_{1}}{n_{0}+n_{1}}\right\rangle
$$

where $n_{1}$ is the number of neighbors with speed $v_{1}$ and $n_{0}$ is the number of neighbors with speed $v_{0}$. With this definition $\gamma$ will vary in the range $[0,1]$. For $\gamma=1$, cells with speed $v_{0}$ will have only neighbors with speed $v_{1}$, and for $\gamma=0$ cells with speed $v_{0}$ will be surrounded only by neighbors with speed $v_{0}$. So $\gamma$ is directly related to the length of the interface formed among cells of different kind.

\section{RESULTS}

We present here two-dimensional simulation samples with $N$ cells. While exploring the parameter space, we use $N=2000$; detailed segregation evolution simulations are performed with $N=8000$ cells. Since the number of cells is fixed in each simulation, we initially explored three different proportions of slower $\left(v_{0}\right)$ to faster $\left(v_{1}\right)$ cells: (a) $1: 3$, (b) $1: 1$, (c) $3: 1$. At $t=0$, cells are randomly distributed in a circular area of radius $R=r_{e} \sqrt{N}$. The value used for the force parameter $\beta$ throughout this work is 0.55 . With that $\beta$ value, an isolated group of cells with speed $v_{0}$ would be in the solid phase close to the liquid transition, while a group of cells with speed $3 v_{0}$ would be in the liquid phase. Figure 1 shows the asymptotic state for these cases at $\alpha=0$. Case 1 shows complete segregation. Case 2 presents partial segregation with the faster cells caged in a ring formed by the slower ones. No segregation is found in case 3 .

In the following we study in detail case 1 since it presents complete segregation. Note that due to the initial random configuration and the fixed proportion among cell kinds, we expect $\gamma=0.75$ at $t=0$ in case 1 . 

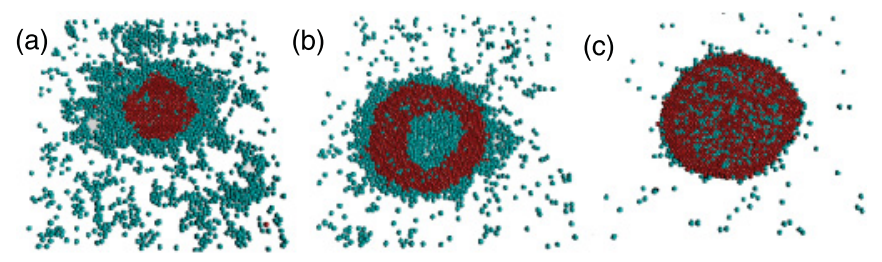

FIG. 1. (Color online) Snapshot of the asymptotic configuration of cells for three different proportions of slower to faster cells: (a) $1: 3$; (b) $1: 1$; (c) $3: 1$. In all cases, $N=2000, \delta=4, \alpha=0$.

In the absence of coordinated motion $(\alpha=0)$, models $\mathrm{a}, \mathrm{b}$, and $\mathrm{c}$ are the same, producing equivalent results. The difference among cell types is due only to their speed. A first simulation within this condition is shown in Fig. 2 for $\delta=4$. This plot shows that, discarded a transient of $10^{4}$ time steps, the evolution of the order parameter is well described by a power law with exponent $\lambda \sim-0.22( \pm 0.02)$. This figure also shows the same trend when a different total cell number is used.

As can be noted in Fig. 3, a large fraction of cells disperse away from the main cell cluster, mainly the faster cells. In order to avoid taking into account these cells in the computation of the order parameter, an algorithm for cluster identification has been used to count only clusters with more than 10 cells. The curve for the order parameter evolution found (not shown) is the same, only rescaled to a constant value below $(\gamma \rightarrow$ $\gamma-0.05)$.

Four system snapshots presenting cell sorting for a system with $N=8000$ cells and $\alpha=0$ are shown in Fig. 3. In the first picture [Fig. 3(a)] the initial random configuration used is shown. Figure 3(b) shows the formation of small clusters at $t=10^{5}$. These first clusters grow and fuse as shown in [Fig. 3(c)] for $t=10^{7}$. Finally they join in a single cluster, finishing the cell sorting at $t=10^{8}$ [Fig. 3(d)].

Now we consider the cases with $\alpha \neq 0$, that is, the cases $\mathrm{a}, \mathrm{b}$, and c, described above, where the velocities of the neighboring cells also influence each cell. Since our model includes cells with two velocities, differently from the standard Vicsek model [13], we do not expect the transition to coordinated motion to

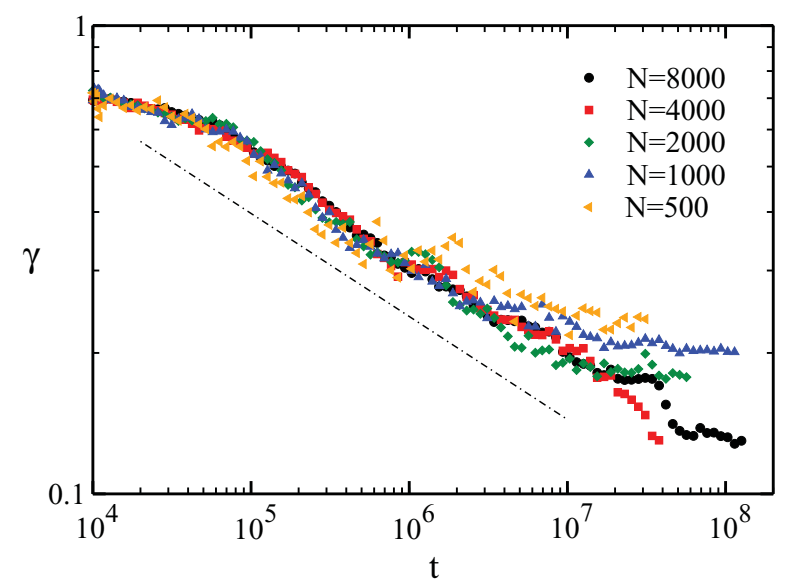

FIG. 2. (Color online) Evolution of the segregation order parameter. The ratio between cell speeds is $\delta=4$. The straight line indicates a power law with exponent $\lambda \sim-0.22( \pm 0.02)$. (a)

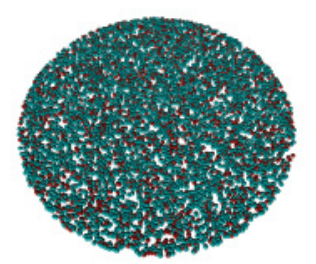

(b)
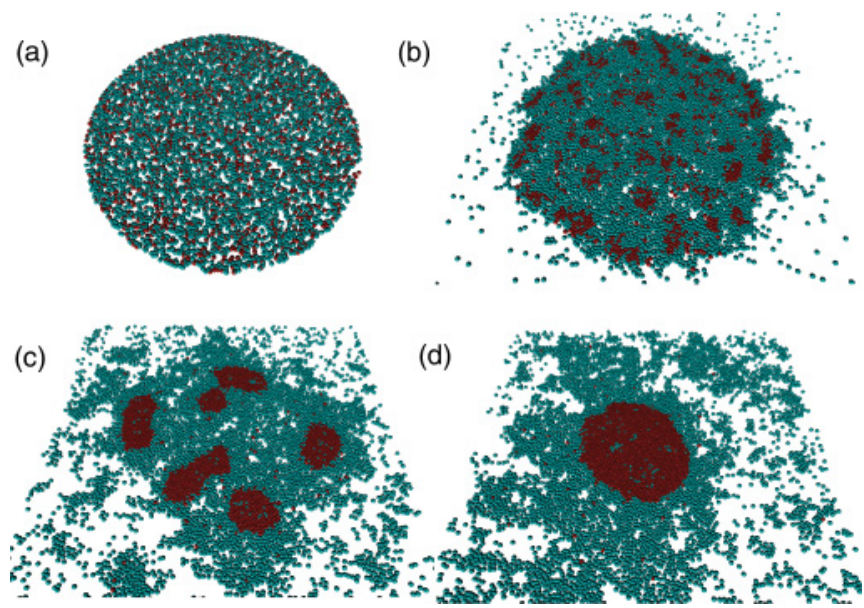

(d)

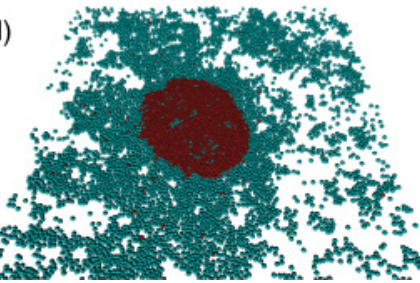

FIG. 3. (Color online) System snapshots at $t=0$ (a), $t=10^{5}$ (b), $t=10^{7}(\mathrm{c})$, and $t=10^{8}(\mathrm{~d})$. In all cases $N=8000, \delta=4$, and $\alpha=0$.

happen at the same $\alpha$ values for each cell kind. The diagrams in Fig. 4 indicate parameter regions where different combinations of collective motion happen for each cell kind: (i) $V_{0} \sim V_{1} \sim$ 0 , (ii) $V_{0} \sim V_{1} \neq 0$, and (iii) $V_{0} \neq V_{1} \neq 0$, where $V_{i}$ is the center of mass velocity for the ensemble of cells of kind $i$. Since in case iii the center-of-mass velocity associated to the two cell kinds is different, all model variants $(a, b, c)$ will present tissue separation, so we will not explore parameters in this region.

The shaded regions in Figs. 4(a), 4(b), and 4(c) indicate parameter ranges with segregation. The difference in shades are associated to the specific time dependency fitted: logarithmic or power law. This is detailed below. It can be noted that in model a the segregation region is narrower when compared to models b and c; this reflects the fact that the aggregate loses cohesion at moderate $\alpha$ values (close to the continuous line in the interval $7 v_{0}<\alpha<14 v_{0}$ ). Cell aggregates in models $\mathrm{b}$ and $\mathrm{c}$ also lose cohesion but at larger $\alpha$ values; this is indicated by the straight dashed line close to region ii of Figs. 4(b) and 4(c).

The segregation results are presented in Figs. 5(a), 5(b), and 5(c). First, it should be noted that the system presents a transient behavior at short times that extends to $t \sim 10^{5}$. In all models, when $\delta=3.0$ a logarithmic dependency in time is a good fit, but a power-law fit also could be a good candidate. For $\delta=3.5$ and $\delta=4.0$ a power law with exponent $\lambda \approx-0.22 \pm 0.02$ was found. Both logarithmic and power-law time dependencies are represented in the figures as dashed lines. A Levenberg-Marquardt algorithm has been used in the fit, and the errors associated to the fitting parameters are below $10 \%$. The cases of larger asymptotic order parameter, that is, less segregated systems, are usually associated to logarithmic evolution, suggesting that this could be a transitory behavior in larger systems.

Comparing these figures it can be noted that the velocity difference is the most important factor for the segregation. Parameter $\alpha$ also improves segregation in all models, but is more relevant in model a and less in model c. In any case, it is clear that both parameters $\alpha$ and $\delta$ shorten the initial transient behavior. 


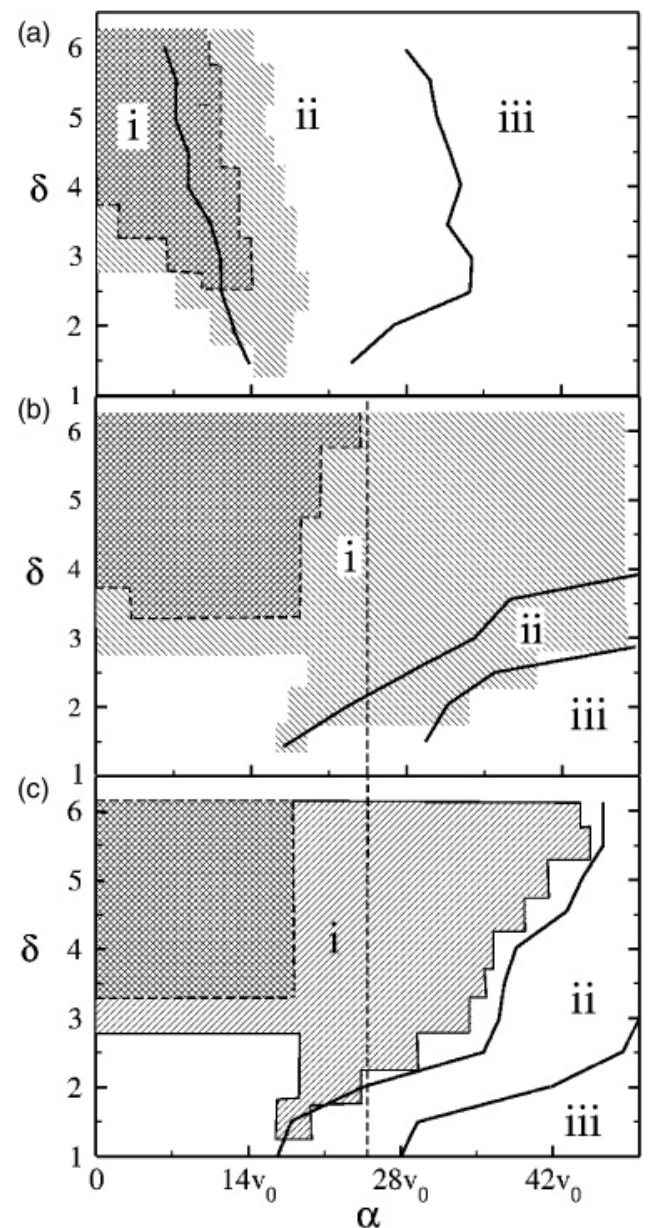

FIG. 4. Speed ratio $\delta$ vs parameter of coordinated movement $\alpha$ in models $\mathrm{a}, \mathrm{b}$, and $\mathrm{c}$. The curves separate regions of different center-ofmass behavior. Each point in this parameter space is generated using $N=2000$ particles. See text for details.

\section{DISCUSSION AND CONCLUSION}

To summarize, we have studied the effects of cell velocity differences as a segregation mechanism. The system used is an adaptation of Vicsek's model allowing us to assess separately different physical contributions such as cell adhesion, coordinated cell movement, cell membrane fluctuations, and cell speed differences. Three distinct models were introduced to account for the effect of velocity differences on the coordinated movement. We have found that using an adequate proportion of fast and slow cells and large enough velocities rate $(\delta)$, the segregation may present a power-law behavior even in the absence of coordinated motion $(\alpha=0)$. For $\alpha \neq 0$ the segregation is faster; however, above some value ( $\alpha \sim 14$ for model a and $\alpha \sim 25$ for models $\mathrm{b}$ and $\mathrm{c})$, the aggregate loses cohesion, which also happens for $\delta$ values above $\sim 6.0$. As found in previous simulations under the differential adhesion hypothesis, both power [11] and logarithmic laws [8] may be observed during segregation. We have found these regimes in models $\mathrm{a}, \mathrm{b}$, and $\mathrm{c}$. In the case of a power law, the exponents are in the range $-0.18 \gtrsim \lambda \gtrsim-0.22$. Similar behavior was found for a wide range of the total number of cells (500 to 8000).

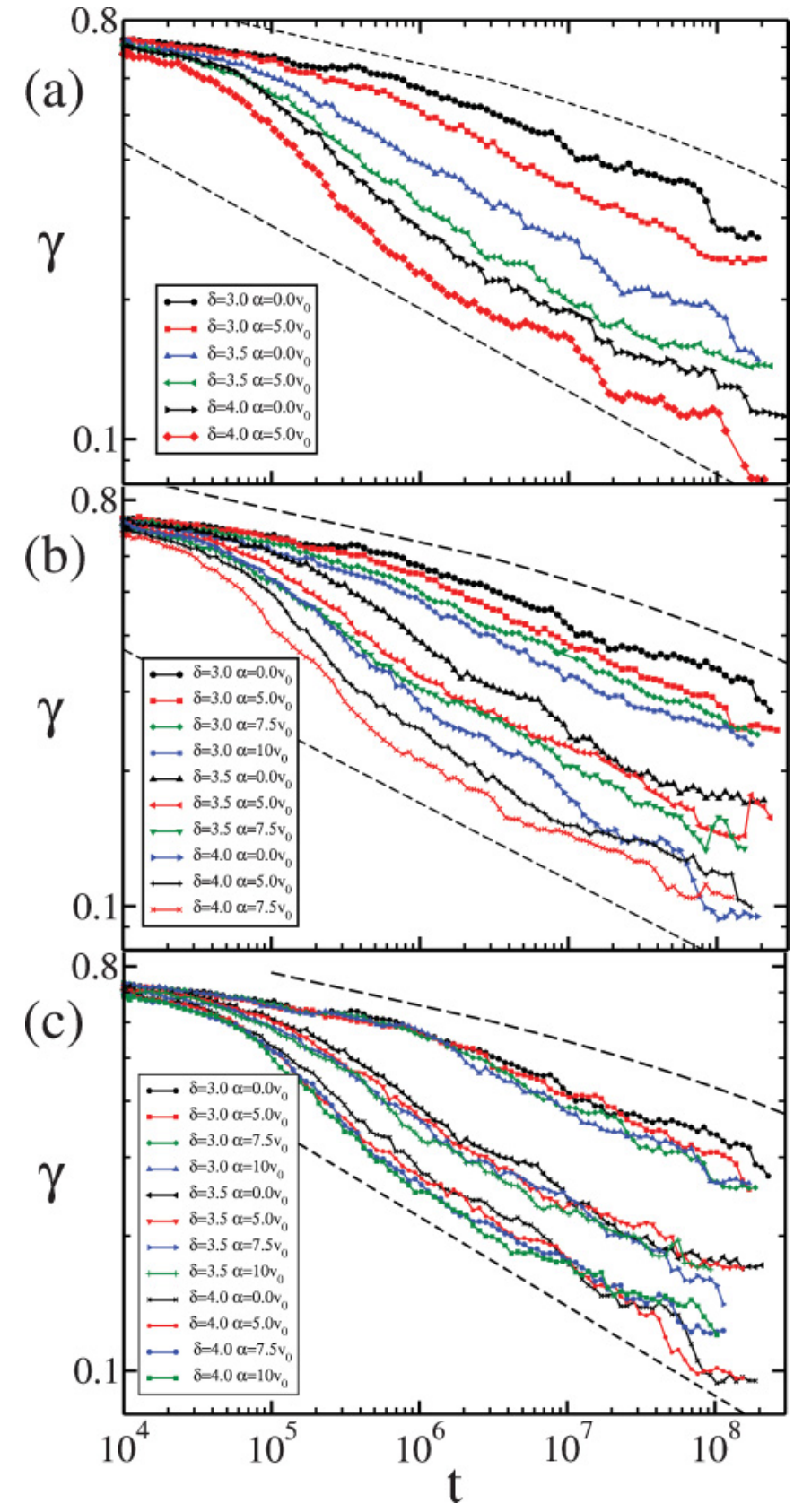

FIG. 5. (Color online) Evolution of the segregation parameter $\gamma$ in models $\mathrm{a}, \mathrm{b}$, and c. Values for parameters $\delta$ and $\alpha$ are indicated in the figure. The system contains $N=8000$ particles. Dotted lines represent logarithmic (up) and power-law (down) fits.

With these results we conclude that the differential speed is a possible physical mechanism to generate cell sorting. The physical interpretation within the boids model used is simple: Since all cells are under equal adhesion forces but have different speeds, the typical adhesion time of two cells [19] depends on the average motility of these cells. So two slow cells will tend to have their positions and velocities more correlated than a fast cell and a slow one, and these latter are more correlated than two fast cells. The coordinated motion helps cell sorting to increase speed and reduce the differences in the speeds necessary to achieve segregation. It should be stressed that when using the 1:3 proportion, the slower cells 
remained surrounded by the faster ones. Slower cells surrounding faster ones were partially found with the 1:1 proportion, but segregation was never complete in this case. On the other hand, in the experiment by Jones et al. [7], the opposite was reported: Slower cells are mostly surrounding the faster ones. In order to explain these results, besides the difference in speed, they also introduced the concept of recognition, in which cells of the same tissue stick to each other better than with cells of different tissues. This is somewhat different from the differential cell adhesion hypothesis [6,20,21]. From our work we can conclude that, despite producing cell segregation, velocity differences alone cannot account for the results of Jones et al. Further simulation work is necessary to decide whether the recognition mechanism together with velocity differences can translate the experimental findings.

\section{ACKNOWLEDGMENTS}

We thank the Brazilian agencies Capes, CNPq, and FAPERGS for financial support.
[1] M. J. Ratcliff, Isis 95, 255 (2004).

[2] M. Kücken, J. Soriano, P. A. Pullarkat, A. Ott, and E. M. Nicola, Biophys. J. 95, 978 (2008).

[3] P. Schiefferdecker, Z. Wiss. Mikr. 3, 483 (1886).

[4] V. H. Wilson, J. Exp. Zool. 5, 245 (1907)

[5] J. Holtfreter, Rev. Can. Biol. 3, 220 (1944).

[6] M. S. Steinberg, Science 141, 401 (1963).

[7] B. M. Jones, P. M. Evans, and D. A. Lee, Exp. Cell. Res. 180, 287 (1989).

[8] F. Graner and J. A. Glazier, Phys. Rev. Lett. 69, 2013 (1992).

[9] N. B. Ouchi, J. A. Glazier, J.-P. Rieu, A. Upadhyaya, and Y. Sawada, Physica A 329, 451 (2003).

[10] M. Alber, N. Chen, P. M. Lushnikov, and S. A. Newman, Phys. Rev. Lett. 99, 168102 (2007).

[11] J. M. Belmonte, G. L. Thomas, L. G. Brunnet, R. M. C. de Almeida, and H. Chaté, Phys. Rev. Lett. 100, 248702 (2008).
[12] C. W. Reynolds, Computer Graphics 21, 25 (1987).

[13] T. Vicsek, A. Czirók, E. Ben-Jacob, I. Cohen, and O. Shochet, Phys. Rev. Lett. 75, 1226 (1995).

[14] G. Grégoire, H. Chaté, and Y. Tu, Physica D 181, 157 (2003).

[15] H. Haga, C. Irahara, R. Kobayashi, T. Nakagaki, and K. Kawabata, Biophys. J. 88, 2250 (2005).

[16] A. Szabó, R. Ünnep, E. Méhes, W. O. Twal, W. S. Argraves, Y. Cao, and A. Czirók, Phys. Biol. 7, 046007 (2010).

[17] J. P. Rieu and Y. Sawada, Eur. Phys. J. B 27, 167 (2002).

[18] J. P. Rieu, A. Upadhyaya, J. A. Glazier, N. B. Ouchi, and Y. Sawada, Biophys. J. 79, 1903 (2000).

[19] A. S. Curtis, Exp. Cell Res. (Suppl.) 8, 107 (1961).

[20] M. S. Steinberg, Dev. Biol. 180, 377 (1996).

[21] R. A. Foty and M. S. Steinberg, Dev. Biol. 278, 255 (2005). 